09

\title{
Физика интерференции квантовых частиц
}

\author{
() С.В. Ганцевич, В.Л. Гуревич
}

Физико-технический институт им. А.Ф. Иофрфе РАН, Санкт-Петербург, Россия

E-mail: sergei.elur@mail.ioffe.ru

Поступила в Редакцию 6 июня 2019 г.

В окончательной редакции 6 июня 2019 г.

Принята к публикации 10 июня 2019 г.

\begin{abstract}
Квантовый язык бра + кет, введенный ранее авторами для объяснения квантовой корреляции на макроскопических расстояниях, позволяет понять физический механизм интерференции отдельных квантовых частиц без представления о превращении волны в частицу и наоборот, а также избежать утверждений о ретрокаузальности, нелокальности, мгновенном взаимодействии и других подобных явлений, якобы присущих квантовой механике. В рамках бра + кет подхода рассмотрены опыты с двумя и тремя щелями, опыты с отложенным выбором и некоторые более сложные интерференционные опыты с переменными параметрами, результаты которых вызвали оживленную многолетнюю дискуссию с противоположными выводами о траекториях частиц в интерферометрах.
\end{abstract}

Ключевые слова: квантовая механика, интерференция, волны и частицы.

DOI: 10.21883/FTT.2019.11.48418.510

\section{1. Введение}

Интерференция потоков квантовых частиц, представленных набором волновых функций - квантовых волн, вполне аналогична интерференции соответствующих классических волн. Однако волновые функции являются не физическими волнами, а волнами вероятности. При уменьшении интенсивности потока процесс интерференции распадается на совокупность дискретных событий, вызванных отдельными частицами. В опытах с двумя щелями, через которые одна за другой проходят отдельные частицы, характерная для волн интерференционная картина восстанавливается в виде набора огромного числа точек экрана, которые соответствуют попаданию отдельных частиц [1]. Таким образом, опыт показывает, что отдельные квантовые частицы интерферируют сами с собой, до этого проходя одновременно по разным путям. Такое поведение не имеет какого-либо рационального объяснения на языке точечных частиц, являясь, по словам Фейнмана, главной тайной квантовой механики.

Принято считать, что квантовая частица может вести себя как волна или как точечная корпускула. В физической литературе квантовый объект, проявляющий свойства классической волны и классической корпускулы, следуя Эддингтону, часто называют волницей (wavicle). Поведение волницы зависит от условий опыта. Так, попадая на сплошной экран, волница ведет себя как точечная корпускула, а перед экраном с двумя щелями она ведет себя как классическая волна, проходя через обе щели и тем самым создавая условия для интерференции. По Бору волновая и корпускулярная природы волницы дополнительны друг другу и не могут проявляться одновременно. Этот корпускулярноволновой дуализм вызывал и вызывает многочисленные попытки раскрыть физический механизм, ответственный за поведение волницы, которое в рамках канонической (копенгагенской) интерпретации квантовой механики считается априорно заданным и не подлежащим какомулибо уточнению. В свое время Уилер [2] предложил мысленный опыт с т.н. отложенным выбором, когда условия эксперимента меняются в процессе его проведения. C усовершенствованием техники эксперимента такие опыты („delayed choice experiments“) были осуществлены [3,4]. Их интерпретации посвящена обширная литература. Поскольку интерференция исчезает, если известен путь волницы в интерферометре, были осуществлены опыты по „стиранию“ этой информации и восстановлению интерференционной картины. Все опыты подобного рода подтвердили предсказания квантовой механики, не добавив ничего принципиально нового [5].

Были предприняты различные попытки заменить интерпретацию дуализма волна-частица по Бору другими представлениями. Все они содержат спорные элементы и не получили до сих пор заметного распространения.

В последние годы были проведены опыты с волницами (фотонами и нейтронами) в сложных интерферометрах с медленно меняющимися параметрами. Предполагалось, что по показаниям чувствительного к подобным изменениям детектора можно определить путь волницы в интерферометре до ее регистрации детектором. В этих опытах волницы (фотоны в [6] и нейтроны в [7]) шли по трем путям, причем два из них имели общие вход и выход. Из-за этой особенности можно было использовать дополнительную интерференцию внутри ветвления до детектора. Как оказалась, эта интерференция существенно влияла на показания детектора.

Для интерпретации своих наблюдений авторы [6] использовали представление о двух квантовых состояниях, 
развивающихся по ходу времени и против него (TSVF two states vector formalism). Результатом применения этого подхода к анализу наблюдений стало утверждение о разрывности траекторий фотонов в составном интерферометре Маха-Зендера, который использовался в их опытах. Представление о разрывных траекториях вызвало ожесточенную критику сторонников более традиционного подхода с различными альтернативными объяснения данных наблюдений (см., например, [8-10]), а также ответные возражения авторов подхода TSVF [11-14].

Заметим, что TSVF-подход, где для описания измерений наблюдаемых физических величин используются две волновые функции (два вектора гильбертова простран-

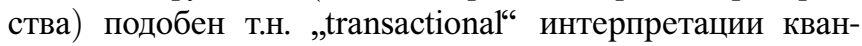
товой механики. Эта интерпретация (TI), развиваемая Крамером и Кастнер (см., например, $[15,16])$ также использует две волновые функции в виде,,волны запроса offer wave“ и „волны подтверждения - confirmation wave“ для описания результата физического процесса, в частности, излучения и поглощения электромагнитных волн - фотонов.

\section{2. Наблюдаемые величины бра + кет функции}

Следует подчеркнуть, что согласно формулам квантовой механики два состояния, две волновые функции или же два вектора гильбертова пространства необходимы для описания любой наблюдаемой физической величины.

В терминах Дирака [17] физическая величина представляется матричным элементом - bracket = $\langle$ bra $|C|$ ket $\rangle$, где оператор наблюдаемой величины $C$ стоит в обкладках из векторов „,bra“ и „ket", которые характеризуют состояние квантовой системы (частицы). Однако, как бра так и кет развиваются по ходу времени, являясь решениями своих уравнений Шредингера. Считать бравектор развивающимся против хода времени нет никаких оснований.

Каноническая интерпретация использует только кетязык, т. е. язык волновых функций. При этом возникают трудности при интерпретации выражений для наблюдаемых физических величин, которые появляются при расчетах, и где стоят одновременно бра и кет функции. Эти трудности, по нашему мнению, носят искусственный характер, отражая неполноту обычно употребляемого кет-языка. Бра+кет язык нашего подхода можно считать уточнением канонической интерпретации в отношении более детальной связи физических представлений и математического аппарата. Ранее мы использовали бра + кет физическую картину для объяснения микроскопической и макроскопической обменной квантовой корреляции $[18,19]$, (см. также [20]). Ниже мы применим бра + кет картину для описания одночастичной квантовой интерференции т.е. для интерференции волницы с собой. Поскольку в этой картине имеется однозначное соответствие между волновым и корпускулярным языком, далее мы будем понимать под бра и кет как амплитуды вероятности (волновые функции или векторы гильбертова пространства), так и описываемые этими амплитудами физические объекты. При этом наблюдаемой физической величине всегда сопоставляется бра + кет пара.

Особенностью математического аппарата квантовой механики является линейность уравнений движения для амплитуд вероятности (бра и кет), так что сумма двух бра-решений также является бра-решением, а сумма кетрешений - кет-решением. В то же время наблюдаемая физическая величина требует билинейной бра + кет комбинации, стоящей в соответствующей скобке Дирака (bracket). Это обстоятельство приводит к появлению двух типов значений наблюдаемых физических величин, за которые ответственны бра + кет пары, составленные из одинаковых или разных по квантовому индексу состояний.

Для обычной интерференции от двух щелей и экрана - детектора мы имеем для зависящей от времени и координат кет-функции

$$
\psi(r, t)=c_{1} \psi_{1}(r) e^{-i \omega_{1} t}+c_{2} \psi_{2}(r) e^{-i \omega_{2} t} .
$$

Здесь $\omega-$ частота, а $c=|c| e^{i \varphi}-$ константа. Аналогичный вид имеет формула для комплексно-сопряженной бра-функции $\psi^{\dagger}(r, t)$. Индексы относятся к двум состояниям (двум щелям или двум путям). (Для краткости векторные значки здесь и далее мы опускаем.) Состояния $|1\rangle$ и $|2\rangle$ считаем собственными функциями гамильтониана, ортогональными и нормированными: $\langle 1 \mid 1\rangle=\langle 2 \mid 2\rangle=1$ и $\langle 1 \mid 2\rangle=\langle 2 \mid 1\rangle=0$.

Наблюдаемой величине - точке экрана с координатой $x$ сопоставим оператор положения $\delta(r-x)$, для которого значение наблюдаемой равно

$$
\begin{aligned}
U(x, t)= & \langle\psi(r)|\delta(r-x)| \psi(r)\rangle=\left|c_{1}\right|^{2}\left|\psi_{1}(x)\right|^{2} \\
& +\left.\left|c_{2}\right|^{2}|| \psi_{2}(x)\right|^{2}+c_{1}^{\dagger} c_{2} \psi_{1}^{\dagger}(x) \psi_{2}(x) e^{i\left(\omega_{1}-\omega_{2}\right) t} \\
& +c_{2}^{\dagger} c_{1} \psi_{2}^{\dagger}(x) \psi_{1}(x) e^{i\left(\omega_{2}-\omega_{1}\right) t}
\end{aligned}
$$

Как мы видим, значение наблюдаемой распадается на два различных по своим свойствам вклада. Первый вклад, представленный суммой квадратов модулей волновых функций в формуле (2), не зависит от фаз и соответствует случаю, когда бра и кет проходят через одну и ту же щель. В этом случае никакой интерференции не наблюдается. Например, для однородного потока фотонов и одинаковых щелей этот вклад соответствует равномерному освещению экрана. Для разных щелей этот фон может быть неоднородным.

Второй вклад в формуле (2) соответствует случаю, когда бра и кет проходят через разные щели. В этом случае разность фаз бра и кет в точке наблюдения оказывается существенной, и именно она ответственна за возникновение интерференции. 
Если проинтегрировать по координатам экрана, то интерференционный вклад исчезает

$$
\bar{U}=\left|c_{1}\right|^{2}+\left|c_{2}\right|^{2} \equiv F_{1}+F_{2}=F
$$

Здесь $F_{1}$ и $F_{2}$ характеризуют интенсивности потоков бра + кет пар с одинаковыми индексами, проходящих соответственно через щели 1 и 2 , а $F-$ полную интенсивность потока через обе щели. Для отдельно взятой частицы, которая с достоверностью попадает на экран $F=1$, так что для одинаковых щелей $F_{1}=F_{2}=1 / 2$.

Исчезновение интерференционного вклада согласно формуле (3) показывает, что полная интенсивность или же полное число точек экрана не зависит от интерференционного вклада, который поэтому можно считать знакопеременными флуктуациями над постоянным средним фоном.

Константы $c_{1}$ и $c_{2}$ можно выразить через интенсивности $F_{1}, F_{2}$ и фазы $\varphi_{1}, \varphi_{2}$, что дает: $c_{1}=\sqrt{F_{1}} e^{i \varphi_{1}}$ и $c_{2}=\sqrt{F_{2}} e^{i \varphi_{2}}$.

В этих обозначениях имеем для интерференционного вклада:

$$
\begin{aligned}
\Delta U(x, t) & =\sqrt{F_{1} F_{2}} 2 \operatorname{Re}\left[\psi_{1}^{\dagger}(x, t) \psi_{2}(x, t)\right] \\
& =2 \sqrt{F_{1} F_{2}}\left|\psi_{2}^{\dagger}(x) \psi_{1}(x)\right| \cos \Phi(x, t) .
\end{aligned}
$$

Здесь разность фаз в точке наблюдения равна

$$
\Phi(x, t)=\left(\omega_{2}-\omega_{1}\right) t+\phi_{1}(x)-\phi_{2}(x) .
$$

Фазы $\phi_{1}$ и $\phi_{2}$ есть суммы начальных фаз $\varphi_{1}$ и $\varphi_{2}$ и фаз приобретенных бра и кет при движении в точку $x$. Для случая когерентных волн $\omega_{1}=\omega_{2}$ разность фаз $\Phi$ постоянна во времени и определяется только различными путями бра и кет. Для недостаточно когерентных волн $\Phi$ флуктуирует во времени, так что интерференционная картина нестационарна. При усреднении по большим промежуткам времени она исчезает.

В опытах с отдельными волницами от когерентного источника [1] интерференционная картина возникает как совокупность точек, возникающих на экране-детекторе с разной вероятностью. Эту вероятность, отсчитанную от постоянного фона (3) следует определить по формуле

$$
\cos \Phi=\cos ^{2}(\Phi / 2)-\sin ^{2}(\Phi / 2) .
$$

Здесь $\cos ^{2}(\Phi / 2)$ определяет вероятность конструктивной интерференции (положительного интерференционного вклада), а $\sin ^{2}(\Phi / 2)$ вероятность деструктивной интерференции (отрицательного интерференционного вклада). При изменении разности фаз $\Phi$ от 0 до $\pi$ полностью конструктивная интерференция переходит в полностью деструктивную.

При усреднении по фазам интерференция исчезает, поскольку вероятности конструктивной и деструктивной интерференции оказываются равными

$$
\overline{\cos \Phi}=\overline{\cos ^{2}(\Phi / 2)}-\overline{\sin ^{2}(\Phi / 2)}=1 / 2-1 / 2=0 .
$$

\section{3. Физика интерференции на бра + кет языке}

Обычно объяснение интерференции отдельных квантовых частиц ограничивается словами „проявление корпускулярно-волнового дуализма“ „неклассическое поведение“ и т.п. Принято считать, что наше воображение, воспитанное на повседневном классическом опыте, не может представить себе физический объект, обладающий такими свойствами. Но, как мы видим, использование бра + кет пар в качестве таинственных волниц позволяет легко примирить как волновой, так и корпускулярный язык с формулами и принципами квантовой механики, а также достаточно естественным образом представить физическую картину движения таинственной волницы в виде бра + кет пары корпускул с меняющимися фазами. Такие осциллирующие корпускулы успешно используются для численной симуляции квантово-механических эффектов $[21,22]$.

Волница в виде бра + кет пары обладает естественным размером - характерным расстоянием между бра и кет. Как показывает опыт, обычно оно порядка дебройлевской длины волны соответствующего квантового состояния. Именно на таких масштабах начинает сказываться, например, различие между волновой и геометрической оптикой. Следует также заметить, что „встреча“ бра и кет в измерительном приборе в действительности представляет собой сложный многоступенчатый физический процесс, в котором бра и кет участвуют по отдельности. Пример такого процесса - поглощение фотона атомом с переходом его в возбужденное состояние с последующей химической реакцией и почернением точки на фотопластинке. Другой пример - образование следа квантовой частицы в камере Вильсона.

Согласно правилам квантовой механики бра и кет по отдельности не наблюдаются, так что информация о пути частицы в интерференционном опыте с двумя путями однозначно свидетельствует о том, что бра и кет прошли именно этим путем. Соответственно, в таком случае интерференция невозможна в принципе. Только так следует понимать „стирание информации“ и особенности опытов с отложенным выбором с якобы влиянием измерений на прошлое.

Действие возмущения в виде потенциала $V$ в гамильтониане по теории возмущений описывается матричными элементами этого потенциала, по форме аналогичными матричным элементам для наблюдаемых величин. Они, однако, характеризуют изменение формы волновых функций, т.е. соответствуют переходам бра $\rightarrow$ бра и кет $\rightarrow$ кет. В каждом таком переходе исчезает одно состояние и возникает другое. Таким образом, и здесь нет никаких величин, развивающихся против хода времени. Изменение хода времени и комплексное сопряжение разные процедуры и их не следует путать. Это особенно хорошо видно, если учесть затухание, которое всегда имеется. 
Как мы видим, физика одночастичной интерференции в опыте с двумя щелями на языке бра + кет весьма проста и полностью соответствует математическому аппарату квантовой механики. Примерно таким же образом можно описать интерференцию в простом интерферометре Маха-Зендера. Особено удобен в этом случае интерферометр для электронов, где нужная разность фаз обеспечивается приложенным постоянным потенциалом (см., например, [23]). Постоянный потенциал не вызывает переходов между квантовыми состояниями, но влияет на фазу. В данном случае фаза растет со временем пробега внутри плеча интерферометра как $e V t$, что согласно формулам (5) и (6) меняет характер интерференции.

\section{4. Медленные возмущения при интерференции}

Рассмотрим теперь влияния медленных возмущений на интерференционную картину. Для большей ясности вместо интерферометров удобно взять более простую для описания систему из двух или более щелей. В опытах с тремя щелями [24] было найдено, что интерференционная картина от трех открытых щелей с большой точностью совпадает с наложением картин от двух щелей с закрытой третьей. Это соответствует представлению о бра + кет паре, проходящей при интерференции по двум путям. В опытах с дифракционной решеткой щелей (штрихов) может быть очень много, но при этом полная картина дифракции является наложением всех возможных комбинаций от двух щелей. Дифрагирующая бра + кет пара при этом может расходиться на большие расстояния, соединяясь потом в измерительном устройстве с соответствующим вкладом в значение наблюдаемой физической величины.

Для двух щелей переменными можно взять размеры щелей и связанные с ними начальные фазы. Будем считать, что размеры щелей влияют только на интенсивность потоков квантовых частиц через них, в то время как начальные фазы определяют положения интерференционных полос вместе с фазами, набранными на соответствующих путях. Медленное изменение размеров щелей т.е. величин $F_{1}$ и $F_{2}$ в формулах (3) и (4) будет влиять на яркость интерференционных полос вплоть до их исчезновения (например, при $F_{2} \rightarrow 0$ ). Положение полос останется неизменным, если разность фаз $\Phi$ в точке наблюдения не меняется. Наоборот, при фиксированных интенсивностях, но при медленном изменении этой разности фаз интерференционные полосы будут смещаться при постоянной яркости. Заметим, что изменение фаз бра и кет на одиниковую величину не сказывается на картине интерференции, т.е. фазовая модуляция при прохождении волницы (т.е. пары бра +кет) целиком через одну щель незаметна.

Рассмотрим теперь опыты в случае сложных интерферометров. Использованный в работе [6] двойной интерферометр Маха-Зендера отличается от простого включением в одно из плеч главного (большого) интерферометра дополнительного (малого) интерферометра. При этом возникает расщепленный надвое участок пути с возможностью дополнительной интерференции. На всех путях фотона от источника до детектора установлены вибрирующие на звуковой частоте зеркала. В спектре детектора на выходе устройства могут наблюдаться участки, соответствующие колебаниям зеркал.

При настройке малого интерферометра на конструктивную интерференцию детектор видит все зеркала. Это означает, что фотоны проходят по всем путям. Однако при настройке малого интерферометра на деструктивную интерференцию детектор не видит зеркала на его входе и выходе, но видит два зеркала в его плечах. Именно этот неожиданный результат интерпретируется в работах [6,11-14] как доказательство разрывности траекторий фотонов, которые попадают в малый интерферометр, минуя его вход и выход. Утверждение о разрывности траекторий фотонов в этом опыте вызвало решительные возражения сторонников непрерывности фотонной траектории с альтернативными объяснениями, опирающимися на волновую оптику и стандартную квантовую механику (см., например, [8-10]). Оживленная дискуссия с множеством публикаций на эту тему продолжается до сих пор (см. ссылки в недавней работе [14]). Авторы более поздней похожей по результатам работе [7] с интерференцией нейтронов считали их траектории непрерывными, не поддерживая подход TSVF.

Заметим, что участники дискуссии неявно считают фотон точечной частицей, сопоставляемой волновой функции, не касаясь тем самым „главной тайны квантовой механики“ с одновременным прохождением точечной частицы по двум путям. В результате интерпретация весьма интересных и поучительных экспериментов, посуществу, сводится к обсуждению линейного отклика достаточно сложной квантовой системы на низкочастотные возмущения. При этом становится неясно, зависит ли этот отклик от принципиальных особенностей системы или же от точности их технического воплощения.

Амплитуда волницы при трех возможных путях от источника до детектора равна сумме парциальных амплитуд $\Psi=\psi_{1}+\psi_{2}+\psi_{3}$. Соответственно в детекторе появляются вклады от их комбинаций $\psi_{j}^{\dagger} \psi_{j^{\prime}}$, т. е. от бра и кет, прошедших независимо по $j$ и $j^{\prime}$ путям. Комбинации в виде чистых бра + кет пар $\left|\psi_{j}\right|^{2}$ образуют независящий от фаз фон, тогда как смешанные бра + кет пары при $j \neq j^{\prime}$ зависят от фаз и участвуют в интерференции. Низкочастотные возмущения в экспериментах [6] и [7] создают малые добавки к $\psi_{j}$ и служат маркерами для соответствующих путей бра и кет. Возмущения модулируют амплитуду и фазу проходящей волны, и эта модуляция может обнаруживаться детектором как соответствующая переменная низкочастотная добавка в его спектре. В модели со щелями возмущению соответствует низкочастотное мерцание интерференционных 
полос без их смещения или же смещение без мерцания, если фазовую и амплитудную модуляции можно наблюдать раздельно. В первом порядке по возмущению эти поправки довольно трудно разделить на эксперименте.

В модели с тремя щелями вклад от каждой пары щелей можно найти, закрывая оставшуюся щель, так как полная интерференционная картина практически соответствует сумме картин от двух щелей. В интерферометре детектор соответствует одной точке экрана при щелевой интерференции. Для трехлучевого интерферометра различить вклады от разных пар путей можно, настраивая их на конструктивную или деструктивную интерференцию. При деструктивной интерференции соответствующий вклад должен исчезнуть. Если при этом возмущения не нарушают условия деструктивности, то в спектре детектора исчезают и соответствующие метки. Этот эффект наблюдался в экспериментах [6,7]. При этом также не наблюдались в первом приближении по возмущению метки на общих участках оставшихся двух путей. Как отмечалось выше, этот эффект привел авторов [6] к выводу об отсутствии фотонов на этих участках и, как следствие, к разрывности фотонных траекторий.

\section{5. О траекториях квантовых частиц}

Несмотря на убедительную критику этого вывода (и самого метода TSVF) с позиций волновой оптики и стандартной квантовой механики со стороны всех остальных участников многолетней дискуссии о траекториях фотонов „в прошлом“ (т.е. до момента наблюдения), сам вопрос об их непрерывности или разрывности не столь однозначен.

Луч света непрерывен в рамках геометрической оптики, но раздваивается при интерференции на самостоятельные бра и кет пути, которые могут заметно различаться. Траекториям бра и кет можно сопоставить фейнмановские виртуальные траектории, образующие интеграл по путям (path integral) [25] математически эквивалентный волновой функции. Волновые функции решения уравнения Шредингера непрерывны. Поэтому можно считать бра и кет траектории непрерывными. По бра и кет можно определить положение наблюдаемой частицы и ее скорость. Но эти величины не могут быть найдены одновременно и соответствующие им математические выражения различны. Это обстоятельство позволяет ввести представление о траектории квантовой частицы только в крупномасштабном квазиклассическом приближении. При переходе к более мелким масштабам наблюдаемая траектория раздваивается на отдельные бра и кет траектории, соответствующие бра и кет волновым функциям.

Как мы видим, бра + кет физическая картина позволяет представить потенциально наблюдаемую квантовую частицу - волницу в виде пары локальных или же квазилокальных физических объектов, описываемых волнами вероятности - решениями уравнения Шредингера. Представление волницы в виде бра + кет пары снимает ряд вопросов тупикового характера, которые только на кет-языке оказываются принципиально неразрешимыми в рамках обычной логики и традиционных физических представлений. Однако, что представляют собой физически бра и кет как „корпускулы с фазой“ остается неясным. Для выяснения этого интересного вопроса, как нам кажется, удобно рассматривать физическую систему, допускающую достаточно простую экспериментальную реализацию. Такой системой может служить, например, „большой атом“ - квантовая точка. В этом случае волны вероятности в пространстве оказываются вещественными стоячими волнами и все различие между бра и кет сводится к экспоненциальным множителям $\exp ( \pm i \omega t)$. При этом бра и кет подобны экспоненциальным решениям обычного осциллятора. Вещественные решения такого осциллятора можно связать с колебаниями амплитуды (положением) и ее производной по времени (скоростью). Эти величины могут быть измерены, но не одновременно. Таким образом, экспоненциальное решение описывает комбинацию из дополнительных друг другу наблюдаемых величин, которые не могут быть измерены одновременно. С другой стороны, именно такие величины удовлетворяют дифференциальному уравнению первого порядка по времени, которое необходимо для прямой причинно-следственной связи во времени. Поэтому только бра и кет траектории могут быть, в строгом смысле, непрерывными (и ненаблюдаемыми), тогда как наблюдения за изменением положения и скорости корпускулы окажутся совокупностью чередующихся точек. Эти точки сливаются в квазиклассическом приближении в непрерывную траекторию наблюдаемой частицы. В результате наблюдаемая классическая частица описывается положением и скоростью - двумя независимыми параметрами, которые теперь уже могут сосуществовать.

\section{6. Заключение}

Экспериментальные исследования траекторий квантовых частиц следует проводить с учетом вышеизложенной бра + кет физической картины интерференции. Это позволит при интерпретации экспериментов однозначно связать физические представления и математический аппарат и избежать ненужных искусственных трудностей.

\section{Конфликт интересов}

Авторы заявляют об отсутствии конфликта интересов.

\section{Список литературы}

[1] A. Tonomura. Nature (London), 393, 33 (1998).

[2] J.A. Wheeler. Life Phys. 337, 1 (1998). 
[3] V. Jacques, E. Wu, F. Grosshans, F. Treussart, P. Grangier, A. Aspect, J. Roch. Science 315 (5814), 966 (2007).

[4] A. Peruzzo, P. Shadbolt, N. Brunner, S. Popescu, J. O’Brien. Science, 338 (6107), 637 (2012).

[5] S.P. Walborn, M.O. Terra Cunha, S. Padua, C.H. Monken. Phys. Rev. A 65, 3, 033818 (2002).

[6] H.B. Danan, D. Farfurnik, S. Bar-Ad, L. Vaidman. Phys. Rev. Lett. 111, 240402 (2013).

[7] H. Geppert-Kleinrath, T. Denkmayr, S. Sponar, H. Lemmel, T. Jenke, Y. Hasegawa. Phys. Rev. A 97, 052111 (2018).

[8] D. Sokolovski. Phys. Rev. A 97, 046102 (2018).

[9] R. Griffiths. Phys. Rev. A 94, 032115 (2016).

[10] Г.Н. Николаев. Письма в ЖЭТФ 105, 3, 136 (2017).

[11] L. Vaidman. Phys. Rev. A 88, 046103 (2013).

[12] L. Vaidman. Phys. Rev. A 93, 36103 (2016).

[13] L. Vaidman. Phys. Rev. A 95, 066101 (2017).

[14] L. Vaidman. Письма в ЖЭТФ 105, 7, ... (2017).

[15] Y. Cramer. Rev. Mod. Phys. 97, 052111 (1994).

[16] R. Kastner. Foudation Phys. 43, 8, 1094 (2012).

[17] П.А.М. Дирак. Принципы квантовой механики. Физматгиз, М. (1960). Гл. 1.

[18] S.V. Gantsevich, V.L. Gurevichю Teor. Phys. 2, 2, 63 (2017).

[19] С.В. Ганцевич, В.Л. Гуревич. ФТТ 60, 1, 5 (2018).

[20] S.V. Gantsevich, V.L. Gurevich. arXiv 1512.03672, 1609.08427, 1701.00448.

[21] H. De Raedt, K. Michielsen. Ann. Phys. (Berlin) 524, 393 (2012).

[22] H. De Raedt, K. De Raedt, K. Michielsen, K. Keimpema, S. Miyashita. J. Comp. Theor. Nanosci. 4, 957 (2007).

[23] Е.Л.Н. Фейнберг. УФН 78, 1, 136 (1962).

[24] U. Sinha, C. Couteau, T. Jennewein, R. Laflamme, G. Weihs. Science 329, 418 (2010).

[25] Р. Фейнман, А. Хибс. Квантовая механика и интегралы по траекториям. Мир, М. (1968).

Редактор Т.Н. Василевская 